\title{
Teores de chumbo em suplementos minerais comercializados no Estado de Mato Grosso do Sul*
}

\author{
Lead level in mineral salt commercialized in the State of \\ Mato Grosso do Sul, Brazil* \\ Wilmar Sachetin Marçal1 ${ }^{1}$ Laurenil Gaste ${ }^{1}$ Marcos Roberto \\ Lopes do Nascimento $^{2}$ Helenara Souza de Oliveira ${ }^{3}$
}

\section{- NOTA -}

\section{RESUMO}

Para se proceder a uma avaliação de misturas minerais, foi realizada uma pesquisa em diferentes formulações, dentre as mais comercializadas no estado de Mato Grosso do Sul, visando a averiguar a presença de metais pesados. O primeiro elemento investigado foi o chumbo, considerado um xenobiótico de grande risco a saúde dos animais. $O$ elemento foi determinado pela técnica de espectrofotometria de emissão atômica. De 19 amostras analisadas, 11 tiveram valores superiores aos $10 \mathrm{ppm}$ recomendado como máximo valor aceitável pelo NATIONAL RESEARCH COUNCIL (1980). Os teores oscilaram entre 1,7 e 460ppm e o maior resultado foi 460 ppm, encontrado numa amostra colhida no municipio de Paranaiba. Os resultados demonstram ser necessário o monitoramento junto aos fabricantes, pois algumas misturas minerais podem ser eminentemente perigosas aos ruminantes.

Palavras-chave: avaliação, chumbo, sal mineral, bovinos.

\section{ABSTRACT}

Lead concentrations in mineral salt sold in the State of Mato Grosso do Sul, Brazil were measured. Lead was determined by atomic absorption spectrometry. Eleven out of 19 mineral mixtures samples presented lead levels higher than 30ppm, the highest tolerated level according to the NATIONAL RESEARCH COUNCIL (1980). Lead concentrations ranged from 1.7 to $460 \mathrm{ppm}$ with the highest found in a sample commercialized in Paranaiba. These findings show the necessity for careful industrial monitoring because some mineral mixtures contain sufficient lead to cause toxicity.

Key words: evaluation, lead, mineral salt,cattle.

\section{INTRODUÇÃO}

O Estado de Mato Grosso do Sul detém o maior efetivo de bovinos do Brasil, com 21.421.567 de cabeças em seu rebanho (IBGE, 2001), a maioria em criação extensiva. De modo direto, a comercialização de sal mineralizado para o consumo animal, naquele Estado, representa uma significativa parcela de dividendos nos agronegócios. Por esse motivo, as indústrias produtoras e/ou misturadoras, visando baratear custos, para ganhar mercado e garantir suas vendas, utilizam fontes de matérias-primas escolhidas pelo preço mais acessível, inclusive por importação.

Este aspecto de preço e qualidade é uma constante preocupação entre nutricionistas, clínicos veterinários e técnicos voltados à saúde e produção animal, pois se acredita que algumas novas formulações minerais possam estar contaminadas por elementos tóxicos, sobretudo metais pesados e substâncias radioativas.

Do ponto de vista econômico, vários autores destacam que os metais pesados, em particular o chumbo, quando presente em suplementos alimentares para animais, podem causar alterações orgânicas importantes, modificando a performance dos animais (LOBÃO 1977; MALETTO 1986; SILVA 1993; ASSOCIATION OF AMERICAN FEED CONTROL OFFICIALS INCORPORATED 2001), podendo

*Trabalho realizado com auxílio financeiro da CPG-UEL, através do Projeto Xenobióticos.

${ }^{1}$ Professor Associado do Departamento de Clínicas Veterinárias da Universidade Estadual de Londrina (UEL), CP 6001, 86020-130,

Londrina-PR. E-mail: wilmar@uel.brAutor para correspondência.

${ }^{2}$ Químico, CNEN (Comissão Nacional de Energia Nuclear).

3 Médico Veterinário, Pós-graduando na UEL. 
acarretar significativas alterações no sistema reprodutivo dos bovinos (McDOWELL, 1985), inclusive abortamento (STUART \& OEHME 1982; McDOWELL 1985; MARÇAL et al., 2001).

Neste aspecto, o objetivo da presente pesquisa foi investigar a presença de xenobióticos em diferentes suplementos minerais mais vendidos no estado de Mato Grosso do Sul, buscando, através de análises laboratoriais específicas, quantificar contaminantes que possam estar agregados aos elementos minerais nas formulações preparadas para alimentação animal.

\section{MATERIAL E MÉTODOS}

As amostras de sal mineral foram colhidas diretamente do estoque disponível em estabelecimentos comerciais (08 amostras), ou de algumas fazendas colaboradoras (11 amostras). Foram colhidas marcas mais comercializadas nos maiores centros pecuários do Estado. As amostras foram acondicionadas em recipientes de plástico transparente, previamente identificadas, com aproximadamente 200 gramas de cada diferente marca de produto. As análises foram efetuadas no Laboratório da Comissão Nacional de Energia Nuclear (CNEN/Poços de Caldas - MG). As contraamostras foram mantidas armazenadas para salvaguardar qualquer necessidade de repetição de análise.

As amostras foram secas à $110^{\circ} \mathrm{C}$, por duas horas, e, em seguida, solubilizadas com ácidos nítrico, perclórico e fluorídrico. O chumbo foi determinado em aparelho de espectrometria de absorção atômica (Varian, modelo 220 FS). Para teores abaixo de $10 \mathrm{ppm}$, o chumbo foi separado da amostra por extração com pirrolidina carbamato de amônia (APDC) p.a. em pH 2,3 $\pm 0,1$, segundo manual da AMERICAN SOCIETY FOR TESTING AND MATERIALS (1980) e a técnica de EATON et al. (1995). O limite mínimo de determinação do método é de $1,5 \mathrm{mg} \mathrm{kg}^{-1}$.

\section{RESULTADOS}

A análise estatística foi feita com o SAS/ BASIC Program (SAS PROCEDURES GUIDE, 1996). Os valores encontrados são apresentados na tabela 1 .

\section{DISCUSSÃO}

Os resultados destacados, na tabela 1 , demonstram que, em 11 amostras, os valores extrapolam o limite máximo aceitável de 30ppm atribuído pelo NATIONAL RESEARCH COUNCIL (1980).

Nestas investigações sobre o chumbo, utilizaram-se as formulações minerais já misturadas, porque não foi possível separar as matérias-primas para investigar cada um de seus componentes. Portanto, trabalhou-se com as formulações industrializadas prontas. A suspeita maior de que a presença de chumbo na mistura esteja incorporada às fontes de fósforo se dá porque estas representam o maior custo na composição de um sal mineral (SOUSA 1981; ROSA 1989), induzindo os fabricantes a buscála em fontes alternativas mais baratas. A possibilidade de o chumbo estar agregado às fontes de fosfato, como por exemplo nos fosfatos naturais de rocha (AMMERMAN et al., 1977; VIANA 1985; ROSA 1989; CAMPOS NETO 1992) ou no ácido fosfórico importado (BRITO, 1993), certamente, pode tendenciar pesquisas de investigação dirigida, muito embora deva-se lembrar que os xenobióticos podem também ser oriundos de matérias-primas de micro-elementos (CAMPOS NETO \& MARÇAL, 1996). Em consonância, a etapa seguinte deste trabalho terá como finalidade investigar as matérias-primas dessas importantes marcas até agora analisadas.

Há, contudo, outros aspectos nessa questão de xenobióticos e que devem ser lembrados como alerta, já que algumas manifestações dos animais podem ser inaparentes ou silenciosas: os bovinos que consumem sal mineral com teores considerados tóxicos podem ter alterações no sistema reprodutivo, com interferências no ciclo estral das vacas, anestro, aumento no intervalo entre-partos e alterações de desempenho (STUART \& OEHME 1982; McDOWELL 1985; MARACEK et al., 1998). Além disso, a absorção de cálcio pelos animais também pode ser prejudicada pela presença de chumbo em formulações minerais, havendo competição por receptores de absorção (BARTON et al., 1978).

É preciso ressaltar, todavia, que existe a possibilidade de uma formulação mineral contaminada com metais pesados, incluindo o chumbo, comprometer a cadeia trófica alimentar, atingindo, inicialmente, os bovinos e, por conseguinte, o homem, conforme referem MALETTO (1986); JUNQUEIRA (1993); CAMPOS NETO \& MARÇAL (1996); MARÇAL et al. (2001). Este aspecto representa, a curto prazo e em larga escala, riscos a saúde pública pelo consumo de produtos e sub-produtos de origem animal potencialmente comprometidos, ao qual já alertaram MALETTO (1986); ANDRIGUETO et al. (1990); ALLEN (1992); CAMPOS NETO \& MARÇAL (1996); MARÇAL et al. (1998). Espera-se, a partir desses 
Tabela 1 - Concentração de chumbo inorgânico (média e desvio padrão) em diferentes marcas de sal mineral, comercializadas no estado de Mato Grosso do Sul, 2001.

\begin{tabular}{lcc}
\hline $\begin{array}{l}\text { N. }{ }^{\text {da }} \text { amostra } \\
01\end{array}$ & Município & $\begin{array}{c}\text { Valores médios de } \\
\text { chumbo (ppm) }\end{array}$ \\
\hline 02 & Aparecida do Taboado & $127 \pm 6$ \\
03 & Brasilândia & $4,0 \pm 0,6$ \\
04 & Caarapó & $31,0 \pm 1,5$ \\
05 & Campo Grande & $64 \pm 3$ \\
06 & Dourados & $77 \pm 4$ \\
07 & Dourados & $3,9 \pm 0,6$ \\
08 & Inocência & $37,2 \pm 1,9$ \\
09 & Maracaju & $43,8 \pm 2,2$ \\
10 & Naviraí & $32,2 \pm 1,6$ \\
11 & Nova Alvorada do Sul & $3,7 \pm 0,6$ \\
12 & Paranaíba & $4,7 \pm 0,7$ \\
13 & Paranaíba & $460 \pm 12$ \\
14 & Paranaíba & $1,7 \pm 0,3$ \\
15 & Paranaíba & $34,8 \pm 1,7$ \\
16 & Paranaíba & $3,7 \pm 0,2$ \\
17 & Rio Verde & $53,0 \pm 2,6$ \\
18 & Três Lagoas & $11,3 \pm 1,1$ \\
19 & Três Lagoas & $29,4 \pm 1,5$ \\
& Três Lagoas & $33,0 \pm 1,6$ \\
\hline
\end{tabular}

resultados, considerados até mesmo como uma triagem, ser possível rastrear o destino das formulações comprometidas e monitorar a sanidade dos bovinos, colhendo-se material biológico, como sangue e tecidos para análise laboratorial comprobatória. Há, ainda, como ponto crítico e alerta, a eminente contaminação ambiental originada pela defecação dos animais que recebem formulações minerais contaminadas, atingindo solo, vegetação e mananciais hídricos.

Pelas razões apresentadas, é necessário que as indústrias misturadoras de sal mineral zelem pela pureza de matérias-primas de suas formulações, uma vez que o crescimento da comercialização desses produtos impuros pode contribuir para a presença de mais contaminantes na alimentação animal. Com isso, pode-se, infelizmente, atingir o homem-consumidor, originando pontos fortemente negativos no competitivo mercado comercial, sobretudo nas exportações.

Ainda no que concerne à via do agronegócio, merece destacar-se também que o estado de Mato Grosso do Sul, pela expressiva população de bovinos que detém, contribui diretamente para que o país continue sendo o detentor do maior rebanho comercial de bovinos no mundo, conforme refere MARTIN (1993). Esse é um forte motivo para se fazer necessária maior vigilância e adequação prática dos ensaios contínuos de rastreabilidade, assegurando que os produtos e subprodutos de origem animal, brasileiros, sejam de inexorável qualidade e oriundos de inquestionáveis criações.

\section{CONCLUSÕES}

A análise dos resultados da presente pesquisa permite as seguintes conclusões:

somente oito amostras analisadas (42\%) apresentaram resultados inferiores aos 30ppm, e onze amostras (58\%) apresentaram resultados superiores aos 30ppm, que é o limite máximo aceitável;

o maior valor encontrado, ou seja, 460ppm, refere-se a uma formulação mineral comercializada no município de Paranaíba.

\section{AGRADECIMENTOS}

Os autores manifestam agradecimentos à Delegacia do Ministério da Agricultura em Mato Grosso do Sul (SFFA/MS), pelas informações oportunizadas.

\section{REFERÊNCIAS BIBLIOGRÁFICAS}

ALLEN, J.D. Minerals in animal feed. Industrial Minerals, v.292, p.35-39, 1992.

AMERICAN SOCIETY FOR TESTING AND MATERIALS. Water. In: Philadelphia, 1980. p.50-464.

AMMERMAN, C.B. et al. Contaminating elements in mineral supplements and their potential toxicity: a review. Journal of Animal Science, v.44, n.3, p.485-508, 1977.

ANDRIGUETO, J.M. et al. Os princípios nutritivos e suas finalidades. In: _. Nutrição animal. 4.ed. São Paulo : Nobel, 1990. p.189-255.

ASSOCIATION OF AMERICAN FEED CONTROL OFFICIALS INCORPORATED. Official guidelines for contaminant levels permited in mineral feed ingredients. Indiana, 2001. V.19, 352p.

BARTON, J.C.; CONRAD, M.E.; HARRISON, L. Effects of calcium on the absorption and retention of lead. Journal of Laboratory Clinical Medical, v.91, p.366-376, 1978.

BRITO, J. de. Fosfato bicálcico feed grade. Cajati : Serrana, 1993. 17p. (Apostila).

CAMPOS NETO, O. Pesquisa esclarece dúvidas sobre déficit na nutrição animal. O Corte, São Paulo, v.24, p.14-15, 1992.

CAMPOS NETO, O.; MARÇAL W.S. Os fosfatos na nutrição mineral de ruminantes. Revista dos Criadores, São Paulo, n.793, p.8-10, 1996. 
EATON, A.D.; CLESCERI, L.S.; GREENBERG, A.E. Standard methods for the examination of water and wastewater. Washington: APHA, 1995. p. 3-16.

IBGE. Calendário Oficial de Exposições e Feiras Agropecuárias. Ministério da Agricultura e Abastecimento. Sociedade Brasileira de Medicina Veterinária. Efetivo pecuário do estado de Mato Grosso do Sul. Brasília, 2001. 90p.

JUNQUEIRA, O.M. Metais pesados contaminam carne. Avicultura \& Suinocultura Industrial, São Paulo, v.38, p.27-29, 1993.

KANEKO, J. Clinical biochemistry of domestic animals. 4.ed. New York: Academic, 1989. p.239-891.

LOBÃO, A.O. Mineralização de bovinos de corte. In: SIMPÓSIO SOBRE PECUÁRIA DE CORTE, 1977, Presidente Prudente. Anais... Jaboticabal : UNESP, 1977. p.120-135.

MALETTO, S. Correlação da nutrição mineral e a sanidade. In: SEMINÁRIO SOBRE NUTRIÇÃO MINERAL, 1986, São Paulo, SP. Anais... São Paulo : SINDAN, 1986. 38p.

MARACEK, I. et al. Residues of heavy metals in cow reproductive organs and morbidity of cattle in the fallout region of a metallurgical plant. Vet Med - Czech, v.43, n.9, p.283-287, 1998

MARÇAL, W.S.; CAMPOS NETO, O.; NASCIMENTO, M R.L. Valores sangüíneos de chumbo em bovinos Nelore suplementados com sal mineral naturalmente contaminado por chumbo. Ciência Rural, Santa Maria, v.28, n.1, p.5357, 1998.

MARÇAL, W.S. et al. Lead concentration in mineral salt mixtures used in beef cattle food supplementation in Brazil. Veterinarski Arhiv, v.69, n.6, p.349-355, 1999.

MARCAL, W.S. et al. Concentration of lead in mineral salt mixtures used as supplements in cattle food. Experimental and Toxicologic Pathology, v.53, p.7-9, 2001.

MARÇAL, W.S.; TRUNKL, I. Poluição industrial na zona rural: implicações na saúde pública. In: CONGRESSO
BRASILEIRO DE MEDICINA VETERINÁRIA, 23., 1994, Olinda, PE. Anais... Olinda : SBMV, 1994. p.656.

MARTIN, L.C.T. Nutrição mineral de bovinos de corte. 2.ed. São Paulo : Nobel, 1993. 173p.

MAYNARD, L. et al. Elementos inorgânicos e seu metabolismo. In: . Nutrição animal. 3.ed. Rio de Janeiro : Freitas Bastos, 1984. Cap.10, p.260-329.

MCDOWELL, L.R. Nutrition of grazing ruminants in warm climates. Orlando: Academic, 1985. p.182-186.

NATIONAL RESEARCH COUNCIL. Subcommitte on mineral toxicity in animals. Washington DC : National Academic Science, 1980. p.256-276.

ROSA, I.V. Fosfato natural como suplemento de fósforo para bovinos. In: VALLE, E.R. et al. Coletânia de seminários técnicos 1986/88. Campo Grande : Embrapa, 1989. p.59.

SAS, Analyses System. Cary : SAS Institute, 1996. 705p.

SILVA, S. Plano de ação fiscal sobre fosfato de rocha e outros. Brasília : Ministério da Agricultura, do Abastecimento e da Reforma Agrária, 1993. 21p. (Apostila).

SOUSA, J.C. Aspectos da suplementação mineral de bovinos de corte. Campo Grande : Centro Nacional de Pesquisa em Gado de Corte, 1981. p.1-50. (Circular Técnica n.5).

STUART, L.D.; OEHME, F.V. Environmental factors bovine and porcine abortion. Veterinary and Human Toxicology, v.24, p.435-441, 1982.

VIANA, J.A.C. Fontes de sais minerais para bovinos e o desafio de suplementos de fósforo no Brasil. In: SIMPÓSIO SOBRE NUTRIC̃̃̃ DE BOVINOS, 3., 1985, Piracicaba. Anais... Piracicaba : FEALQ, 1985. p. 138 .

VILLEGAS-NAVARRO, A. et al. Determination of lead in paired samples of blood and synovial fluid of bovines. Experimenthal and Toxicologic Pathology, v.45, p.4749, 1993. 